(Aus der Frauenklinik der Universität Heidelberg. Director: Geheimrath von Rosthorn.)

\title{
Beitrag zur Lehre von den Lymphangioendotheliomen des Eierstocks.
}

Fon

Dr. Heinrich Eymer.

(Uierzu Tafel Vul-- VıII, )

Vor ganz kurzer Zeit hat in Hegar's Beiträgen (1908 3. Heft) Gabriel Jung mit vollem Rechte vor übereilter Diagnosenstellung bei endothelialen Eierstocksgeschwülsten gewarnt. Er beschreibt zwei Tumoren, die bei der ersten Besichtigung für Endotheliome gehalten wurden, aber dann bei eingehender Betrachtung sich als sichere Carcinome herausstellten. Auch R. Meyer stellt gelegentlich seiner Abhandlung über die Endotheliome des Uterus die Diagnose dieser Geschwulstform auch in Ovarien als äusserst sohwierig, ja manchmal als differentialdiagnostisch unmöglich hin. Schon daraus geht hervor, dass über die Eigenthümlichkeiten der Endotheliome noch viele Unsicherbeiten herrschen müssen, wenn bis jetzt auch schon ca. 50 sichere Endotheliome der Ovarien beschrieben sein mögen. Dieser Stand der Dinge rechtfertigt die Beschreibung eines Tumors, der höchstwahrseheinlich den Lymphangioendotheliomen zuzurechnen ist. Das Präparat wurde durch Laparotomie an der Heidelberger Universitätsklinik gewonnen. Zur genaueren Untersuchung desselben wurde ich von Herrn Prof. Dr. Schottlaender angeregt. Hjerfür, sowie für die weitgehende Unterstützung bei meiner Arbeit, bin ich aufrichtig dankbar.

Die Krankengeschichte sagt kurz folgendes:

Amalie H. Aufn. 26. IX. 07, unverheirathet, 42 Jahre alt, Familienanamnese belanglos. Menstruirt zum ersten Mal mit 17 Jahren, regelmässig alle 4 Wochen. Daner: 4-5. Tage, Blutverlust nicht stark, keine Schmerzen. Seit einem Jahr Periode nicht mehr ganz regelmässig; 
stärkere Blutungen, Kopfschmerzen dabei. Keine Geburt. Kein Abort. Vor 3 Wochen morgens Uebelkeit, Erbrechen. Seit dieser Zeit fühlt Patientin in der Unterbauchgegend eine harte Geschwulst, die sich angeblich nicht vergrössert hat. Schmerzen links unten im Leib, Schmerzen vor dem Stublgang und bei demselben. Brennende Schmerzen veim Uriniren. Nie Ausfluss. Vor 5 Tagen begann eine $1 \frac{1}{2}$ Tage dauernde Genitalblutung, dabei Schmerzen in der Geschwulstgegend links. Die Leibschmerzen bestehen anfallsweise besonders Nachts. Appetitlosigkeit. Seit 8 Tagen wegen Schwäche arbeitsunfahig.

Status praesens: Grosse Frau mit kräfitigem Knochenbau, mittlerer Ernährungszustand. Nammae klein, flach, Warzen gut entwickelt, beiderseits klares Colostrum. Herz und Lunge gesund. Bauchumfänge: über dem Nabel 70 , in Nabelböhe $771 / 2$, zwischen Nabel und Symphyse $80 \mathrm{~cm}$. Untere Partien des Abdomens etwas druckempfindlich, besonders links. Urin: ohne Befund. Hämogiobin $70 \%$ aach Sahli.

Stat. gyn: Geringes Ekzema intertrigo. Noduli haemorhoidales; Praeputium elitoridis, kleine Labien hypertrophisch. Damm niedrig, muldenförmig. Hymen glatt, rechts ein seichter, frischer Einriss. Zwei Fingerglied hoch hinter dem Introitus flache breite Portio mit quer ovalem Muttermund in der Mittellinie stehend. Das hintere Scheidengewölbe ausgefüllt von zerklüfteten theils weicheu, theils harten papillären höckerigen Massen, die einen Raum von etwa Zweimarkstückgrösse einnehmen. In der Umgebung derselben ein harter wulstiger Rand, der auf die hintere Fläche der Portio übergeht. Der Uterus scheint mit einem rechts vorn hinter der Symphyse liegenden kindskopfgrossen eystischen Tumor zusammenzahängen, lasst sich jedoch nicht isolirt heraustasten. Bewegungen dieses Tumors setzen sich auf die Portio fort. In der linken Bauchseite ein 2 Finger über dẹ Nabel hinaufreichender, theils praller, theils solid sich anfühlender fast kopfgrosser Tumor, der hinter der rechtsseitigen Geschwulst etwa 3 Querfinger über die Mittellinie nach rechts hinüberragt. Parametrien sehr straff, unelastisch, ebenso die Bauchdecken, daher eine genauere bimanuelle Untersuchung nicht möglich.

Diagnose: bilateraler maligner Ovarialtumor mit Durchbruch in die Scheide, oder mit selbständigem Carcinom der Scheide.

28. IX. Abdominale Radicaloperation (Dr. Kermauner). Längsschnitt. Man sieht 2 über kindskopfgrosse cystische Tumoren vou beiden Ovarien ausgehend; blutig-seröser Ascites. Linker Tumor stielgedreht, nekrotisch, in breiter Ausdehnung mit dem Netz und der vorderen Bauchwand verwachsen. Der rechtsseitige bängt im Douglas, lässt sich nur unter Zurücklassung narbiu-brüchigen Gewebes, welches mit der gefühlten Platte im hinteren Scheidengewölbe innig zusammenhängt, luxiren. Abtragung beider Tumoren. Ablösung der Blase schwer. Gewebe sehr straff und zäh. Rechts wird oberhalb des Lreters die Blase eingerissen und genäht. Präparation der Lroteren beiderseits schwer. Abbindung der Utterinae. Beiderseits je ein erbsengrosser runder Knoten aus den Parametrien entiemt, nach aussen vom Ureter gelegen. Der in leichter Retrodeviation befiudliche Uterus lässt sich noch nicht hochzjelien. Entsprechend den Sacrouterinligamenten barte Infiltration bis an die Beckenwand. Abklemmen der Ligamente und des paravaginalen Gewebes. Abtragung des Uterus. Umstechungen. Serosadeckung. Streifen in die Scheide. $600 \mathrm{ccm}$ Kochsal\%. 
29. IX. Bei dreistündigem Katheterismus trocken.

2. X. Heilung pp.

6. X. Seit gestern abend liegt Pat. nass: Vesicovaginalfistel.

19. X. Entlassen. Bei Blasenfüllung mit $250 \mathrm{ccm}$ fliesst die Flüssigkeit aus der Scheide ab. In 10 Wochen zur Fisteloperation wieder bestellt.

27. XII. Neuaufnahme. In der Vagina wenig urinöses Secret. In der Blase $100 \mathrm{ccm}$ vollkommen klarer Urin. Im Grund des Vaginalstumpfs ein stecknadelkopfgrosser Granulationspfropf, aus dem ein feiner Urinstrahl hervordringt. Scheide oben durch eine Quernarbe und mehrere sagittal gestellte Narbenspangen abgeschlossen. Oberes Ende des Vaginalstumpfes unbeweglich fixirt in derben Schwielen. In Zeigefingerhöhe Rectumlumen stark eingeengt durch einen von links her vorstehenden harten, haselnussgrossen Knoten. Entsprechend dem rechten Sacrouterinligament eine fast knochenharte Gewebsiarbe.

Diagnose: Vesicovaginalfistel. Locales Recidiv im retrocervicalen Bindegewebe.

Cystoskopie. Blasenscheidenfistel mit Ventilverschluss, beide Ureteren functioniren. Keine Cystitis.

17. I. 08. Fisteloperation. T-Schnitt zur Freilegung der Blase. Naht der $1 \mathrm{~cm}$ langen Fistelöffnung. Naht des Längsschnittes und der Scheidewand. Es wird aus dem linken Knoten in der Narbe ein Stïck zur Untersuchung excidirt, welches Darmmusculatur enthält. Naht des T-Schnittes.

22. I. Nach Dauerkatheterentfernung und Entfernung des Vaginalstreifens Blase leer. In der Scheide reichlich Koth. Wundfäcbe im Scheidengrund grauweiss belegt. Nach Füllung der Blase geht bei $50 \mathrm{ccm}$ die Spülflüssigkeit im Strom durch die Scheide ab. Bei Rectumfüllung ebenfalls Abfluss per vaginam. Der ins Rectum eingeführte Finger fühlt hoch oben die Communication.

3. II. Wenig Urin durch die Scheide. Kein Kothabgang. Entlassung. Scheide blindsackartig geschlossen. Im Abschluss frische Granulationen, in deren Bereich die Blasenscheidenfistel liegt. Im Blindsack harte höckerige Massen mit der Rectumwand in Verbindung. Ein zwischen diesen Massen vorgeschobener Uteruskatheter wird im Rectum gefühlt. Spülflüssigkeit im dicken Strahl aus der Blase in die Vagina. Ebenso aus dem Rectum in die Vagina.

19. II. Blase bei $400 \mathrm{ccm}$ vollkommen continent, Rectumcommunication nicht zu fühlen, Rectum in Fingerhöhe durch derbe Tumormassen stenosirt. Pat. beschwerdefrei.

Seitdem ist von der Pat. nichts mehr gehört worden.

Makroskopische Beschreibung des in Formol und Alkohol gehärteten Präparates.

Das ganze Präparat besteht aus dem Uterus mit einem $2 \mathrm{~cm}$ langen Scheidenstück und den beiden von der Gebärmutter getrennten Ovarialtumoren, die mit den anscheinend nicht wesentlich veründerten Tuben noch in Zusammenhang stehen. Die Ovarialtumoren sind grösstentheils cystisch, zum kleineren Theile solid kugelig, anuähernd kindskopfgross, der rechte etwas kleiner, mit einigen wenig hervortretenden Buckeln und Höckern versehen. Beide zeigen flächenhaft auftretende braunröth. liche, ins Bläuliche spielende Verfärbungen. 
Die ganze Oberfiache des linken Tumors ist glatt, mit einigen mohr uteriuwärts befindlichen theils grösseren, theils kleineren, flächenluaft : uusgebreiteten flockigen, gelblichen, rauben Auflagerungen von ca. $2 \mathrm{~mm}$ Dicke. Auf dem Durchschnitt zeigen sich im wesentlichen drei Cysten, deren grösste apfelgross, vou zwei etwas kleineren, halbmondförmigen Cysten halbkreisartig umgeben wird, Da, wo sich die drei Cysten treffen, ungefähr in der Mitte des Tumors, ist ein solider Zapfen, auf den die Scheidenwände zuführen. Einige flachere, ins Lumen reichende Erhebungen deuten weitere zu Grunde gegnngene Septen an. Die Wände der Cysten sind von sehr ungleicher Dicke. Die innere Oberfläche ist theils glatt, theils mit bröckligem Tumorbelage versehen. Die Cysten communiciren offenbar in Folge secundärer Durchbrechungen an mebreren Stellen miteinander. Der Inhalt ist serös, ganz leicht hämorrhagisch. In den dickeren Stellen der Wand finden sich ferner mehrere bis erbsengrosse Cystchen und einige bis bohnengrosse Einlagerangen eines gelblichen festschwammigen Gewebes von T'mmormassen.

An dem Stiel des linken stielgedrehten Tumors fallen schon makroskopisch die stark erweiterten Gefässe anf.

Der rechte etwas kleinere Tumor (Tafel VII, Fig. 1) ebenfalls rorwiegend cystisch, hat im Grossen Ganzen dieselben Oberflächenverhältuisse wie links. An der Stelle jedoch, wo der Tumor im Douglas und mit dem Uterus verwachsen war, fehlt die glatte Bedeckung auf etwa Kleinhandtellergrösse. An dieser Stelle finden sich geibröthliche, zottige, zierlich zerlïlüftete, bröcklige Massen, die rosettenartig aus dem Cystenimern hervorgewuchert sind. Ausserdem finden sich in der Aussenwand, auf der Tafel VII, Fig. 1 nicht zu sehen, noch mehrere bis wallnussgrosse Cystchen, theils glattwandig, mit durch die Formalinhärtung erstarrter, hellbernsteinfarbiger Masse angefullt, theils vollkommen ausgegassen mit derbschwammigem Tumorgewebe, yom Charakter der oben beschriebenen papillaren Massen. Das Innere wird von einer einzigen, fast kindskopfgrossen Cyste eingenommen, die sich lateralwärts leicht sandulnrartig einschnürt und in eine taubeneigrosse Nebenkammer weiterlïhrt. In der Hanptcyste findet sich an der Stelle, die den Verwachsungen im Douglas entspricht, eine etwa wallnussgrosse papilläre Excrescenz mit warziger Oberfläche, die auf dem Schnitt das mehr glatte Stïtzgeriist und an dieses angesetzt, bröckelige, gelbliche, carunculöse Vassen erkennen lässt.

Die Tuben sind beide ca. $7 \mathrm{~cm}$ lang und liegen den Geschwülsten wie normalen Eierstöcken an, ergeben auch im Grossen Ganzen normale Verhältnisse: beide Fimbrienenden sind offen und unverändert. Indessen finden sich bei beiden, etwa $2 \mathrm{~cm}$ rom abdominalen Ende entfernt, spindelige Auftreibungen bis ca. $1 \frac{1}{2} \mathrm{~cm}$ Durchmesser. Während links die Tube auf dem Schnitt nur fein gefältelte, zierliche Massen, keine compacteren Stellen aufweist, birgt die rechte in ihrem Lumen an der oben bezeichneten Stelle derbere gelb-röthliche, bröckelige Massen.

Der gehärtete Uterus (Sagittalschnitt Tafel VII, Fig. 2), zeigt eine waiz geringe Retrodeviation. Bei einer Länge von $9 \mathrm{~cm}$ und einer Corpusdicke von $3 \mathrm{~cm}$ zeigt er eine Breite ron $5 \mathrm{~cm}$. Die ganze hintere Wand des Collums, die ca. dreimal so dick ist als die vordere, wird eingenommen von einem fast wallnussgrossen Herde eines uterusfremden Gewebes, das etwas weniger feste Consistenz wie dieser hat und die Wand des Cervicaleanals von hinten rechts nach vorne vorbuchtet. An ler Stelle des Tumors ist die maschenartige Zeichnung des Bindegewebes 
verwischt, und man sieht mehrstrahlige Stränge ungefähr von der Mitte der hinteren Collumwand nach dem Cervicalcanal ausstrahlen, und zwar in Gestalt einer aufgeschnittenen Niere, deren Becken in der hinteren Wand und deren Wölbung über der Cervixschleimhaut liegt. Der Tumorknoten geht ohne Grenze in die Umgebung über, scheint aber die Cervicalschleimhaut nirgends zu erreichen. Die Tumormasse greift continuirlich ins paracervicale Gewebe über. Hier stösst sie mit den fest im Douglas verwachsenen papillären Tumormassen des rechten Ovariums zusammen, ohne dass die versehiedenen Antheile getrennt werden könnten. Auch im Corpus ist die vordere Wand etwas dünner als die hintere. Makroskopisch fällt im Corpus das glänzende Reticulum des. Bindegewebes auf. Die ca. $1 \frac{1}{2} \mathrm{~m}$ dicke Schleimhaut ist im Cavum deutlich hervortretend.

Als direkte Fortsetzung des Collumtumors finden sich Geschwulstmassen in der Scheide, und zwar besonders in deren hinterer Wand, von wo aus der Tumor nach rechts und links die Scheide umgreifend weitergewuchert ist, so dass aus den Scheidengewölben derbe, starre Gebilde werden. Das hintere Scheidengewölbe ist fast vollkommen verstrichen und die Tumormassen sind höckerig, papillomatös, bröckelig, in der Scheide zum Vorschein gekommen. Von dem Uebergang zwischen Portio und Scheide gehen die Tumormassen noch eine Strecke links und rechts ins paracervicale resp. paravaginale Gewebe über, um sich dann ohne präcise Abgrenzung hier zu vertieren.

Zum gewonnenen Präparate gehören ausserdem noch zwei grosse derbe Lymphdrüsen, die aus den Parametrien ausserhalb der Ureteren entfernt wurden und auf dem Schnitte etwas markig aussehen.

\section{Mikroskopischer Befund:}

Es wurden mit Eisenhämatoxylin-Eosin und nach Weigert's van Gieson-Methode gefärbte Schnitte untersucht und zwar:

A. aus dem linken Ovarialtumor

1. ein Längsschnitt aus der Wand der grossen Cyste an einer mitteldicken Stelle derselben.

2. ein Längsschnitt aus der Hauptcystenwand, an die ein Septum anstösst.

3. ein Querschnitt durch den Stiel des linken Tumors.

B. aus dem rechten Ovarialtumor

1. ein Längsschnitt aus einer mitteldicken Wandstelle der grossen Cyste.

2. a, b. 2 Längssehnitte aus der Umgebung der kleinen Cysten, die die Wände letzterer mittreffen.

C. und D. ein Querschnitt aus der verdickten Stelle der linken und rechten Tube.

E. ein Sagittalschnitt durch den ganzen Uterus auf einer Anzahl von Serienschnitten. Drüse.

F. Schnitte durch die hintere Wand der Vagina.

G. a und b. Schnitte durch die rechte und linke parametrane

H. Schnitte durch das bej der Fisteloperation excidirte Stück.

A. 1. Schon makroskopisch heben sich auf dem Schnitt kleine, stark mit Hämatoxylin gefärbte Herde hervor, die in das Stroma eingelagert sind. 
Das Stroma ist sehr gefässreich und stark hyalin oder ödematös degenerirt. Mebrere Ruudzellenauhäufungen finden sich vor.

In das Stroma sind einzelne Geschwulstherde eingebettet. Diese weisen als Unterbrechung ihres sonst soliden Baues zablreiche runde, schlauchförmig gestreckte, zipfelig ausgezogene Spalträume auf, die nur von den Tumorzellen begrenzt welden. Den Tumor gegen das Lumen abgrenzend findet sich oft eine Schicht von länglichen Zellen, deren ovale, grosse Kerne in ihrem längsten Durchmesser parallel zur Achse des Spaltraumes orientirt sind. Letztere sind sehr oft leer, nie mit reinem Blute gefüllt; oft bilden Detritus und Geschwulstzellen den Inhalt, d. h. sie liegen meistens mitten im Innern, an den Seiten einen freien Raum lassend. In den kleinen tunden Hohlräumen sieht man oft eigenthümliche ganz homogen geronnene Massen und hie und da weisse Blutkörperchen. Die Tumorzellen sind meist rund oder kubisch, mittelgross mit grossen, stark tingirten, chromatinreichen Kernen; ihre Grösse wechselt.

Auffallend ist der Befund von Riesenzellen, die unten noch weiter geschildert werden sollen. Hier und da, jedoch keineswegs an allen Stellen, ist ein ganz feines, bindegewebiges Stroma, besonders bei der Weigert'schen van Gieson-Methode bis zwischen die einzelnen Zellen verfolgbar.

In der Umgebung des Tumorgewebes sieht man, theits von letzterem ausgehend, theils ganz selbständig, Zeilen von einreihig, Zelle an Zelle liegenden Geschwulstelementen in Gewebsspalten, die spindelig zulaufen und keinerlei Endothelauskleidnng zeigen.

Das Serosaepithel ist an den meisten Stellen nicht erhalten, an den wenigen Orten wo es intact ist, erscheint es einschichtig.

2. Das Stroma gleicht dem oben beschriebenen. Die zahlreichen Gefässe sind stark ektatisch und zeigen meist dünne, atrophische Wandungen. Man sieht öfters Blutgefässe mit hyalin degenerirter. Wand, oder solche, deren Lumen von unregelmässigen, theils dünnen, theils dicken homogen diaphan aussebenden, hyalinen Balken durchzogen ist, zwischen denen sich rothe Blutkörperchen finden. Endlich giebt es Gefässe, deren ganzes Lumen von einer hyalinen Masse ausgefüllt ist. Auch sonst im Stroma finden sich unabhängig von den Blutgefässen hyaline Balken.

An anderen Stellen wieder ist das Stroma zellarm.

Die Tumormassen, die sich an der Innenwand der Cyste und innerhalb des Schnittes in kleinen Herden finden, sind meistens in Zerfall und Nekrose begriffen und zeigen so nichts Charakteristisches.

3. Der Stiel des Tumors besteht aus einer Anzahl von Gefässen aller verschiedensten Kalibers. Alle Arterien zeigen stark hypertrophische Muskelschicht mit gut abgegrenzter innerer Ring- und äusserer Längsmusculatux. Zwischen diesen Elementen findet sich ein derbes vielzelliges Bindegewebe. Auch normale Nerven verschiedener Dicke sind zahlreich zu sehen. Von Geschwulstbildung ist an keiner Stelle etwas nachweisbar.

B. 1. (TafeI VII, Fig.3). Die äussere Serosadeckschicht wird dargestellt durch eine einfache Schicht nicht sehr grosser, platter bis kubischer Zellen mit mittelgrossen runden Kernen, die äusserst stark und ganz homogen gefärbt sind.

Das Stroma zeigt wechselnden Zell- und Gefässreichthum.

In ihm finden sich grössere oder kleinere Alveolen von ovaler bis 
runder Form, die unter Aussparung eines Lumens mit Tumorzellen angefüllt sind. An anderen Stellen sind die Geschwalstzellencomplexe grösser, unregelmässiger, buchtig langgestreckt. Auch das Lumen tritt dann dementsprechend als langgestreckter, oft mannigfach abgeknickter und gebogener Spaltraum auf. Stellenweise ist eine Abgrenzung gegen das Lumen in dem oben beschriebenen Sinne vorhanden. An vielen anderen Stellen dagegen ist die Grenze unscharf und die Zellanhäufung erscheint wie abgebrochen. Auffallend ist an vielen Stellen die deutliche radiäre, pallisadenartige Anordnung der Tumorzellen. Auch brückenartig das Lumen durchkreuzende Stränge, ans 2-3 Zellreihen zusammengesetzt, sind zu beobachten. Hänfig finden sich Riesenzellen zwischen den Tumormassen. Der Inhalt des Lumens ist, soweit ein solcher vorhanden, dem bereits beschriebenen gleichartig.

2. a) Der Haupttheil des ganzen Präparates wird von Geschwulstmassen eingenommen, die langgestreckte Herde bilden. Zwischen ihnen befinden sich lange Streifen eines gefäss- und zellreichen Bindegewebes, das wenig degenerative Veränderungen aufweist. Der Debergang zwischen Tumor- und Stromagewebe ist an verschiedenen Stellen verschieden; an einigen ist durch die Behandlung mit Alkohol eine Retraction des Gewebes eingerreten, so dass sich zwischen Tumor und Stroma eine Dehiscenz gebildet hat. Doch finden sich auch Orte, wo die Rissstelle sich in Tumorgewebe selbst befindet. An den am besten erhaltenen Partieen ist die Grenze zwischen Tumor und Stroma unscharf, die Geschwulstelemente dringen, wenn auch nicht sehr tief, in einer oder doppelter Reihe ins Bindegewebe ein, ganz besonders bei den kleinsten Geschwulstherden.

Ein Stromareticulum zwischen den Zellen ist nicht deutlich ausgesprochen.

Die Zellen des Tumors selbst sind polygonal oder rund und ziemlich verschieden gross. Die Kerne sind rund bis oval, mit deutlichem Chromatingerüste, viele mit deutlichen Kernkörperchen.

Auffällig sind (Tafel VII, Fig. 4 und 5) zunächst Riesenzellen mit 1 oder 2 sehr grossen, etwas stärker gefärbten Kernen. Endlich sind Riesenzellen zu sehen, bei denen $4-15$ verschieden geformte und verschieden grosse Kerne meist dicht beieinander ziemlich in der Mitte der Zelle liegen, von einem wechselnd breiten Protoplasmasaume umgeben.

Kerntheilungsfiguren sind an den Tumorzellen leicht und reichlich nachzuweisen und zwar in den verschiedenen Stadien der Mitose. Ein Vorzugsort läs st sich nicht sicher feststellen.

Ferner fallen Zellen auf, gerade so gross wie die anderen, deien Protoplasma ganz homogen aussieht und sich ganz besonders ausgiebig mit Eosin gefärbt hat. Die Kerne dieser Zellen sind kleiner, und stark und ganz gleichmässig gefärbt. Manchmal findet sich noch ein zweiter kleiner Kerm. Auch sieht man innerhalb der Tumormassen etwa der Grösse einer gewöhnlichen Geschwulstzelle entsprechende Vacuolen, in deren Mitte sich ein offenbar degenerirter Kern mit ganz feinem sternartig geschrumpften Protoplasmasaume befindet.

Hier und da sieht man grosse Zellen mit blass gefärbtem Protoplasma (Tafel VIII, Fig. 6), an deren einem Pole, etwa ein Drittel der Circumferenz eimnehmend, ein länglicher, in der Vlitte etwas aufgetriebener Kern liegt, wodurch ein siegelringartiges Gebilde zu Stande kommt. 
Auffällig sind weiter innerhalb der Geschwulstmasse cirkelrunde Höhlen (Tafel VIII, Fig. 6) die von länglichen, sichelförmigen Zellen, mit etwas ins Lamen vorspringenden Kernen ansgekleidet werden.

b) Auf einem anderen Schnitte findet sich zwischen dem Tumorbelage der grossen Hauptcyste und dem Belage eines der kleinen Cystchen ein mehr oder weniger zellreiches, etwas ödematöses Bindegewebe in dünner Schicbt, die Fasern zu den beiden Cystenwänden parallel laufend.

In diesem Bindegewebe sind deutlich unterscheidbar spindelartig an beiden Enden spitz zulaufende Spalträume, die mit einem eigenen Belage von deutlichen, ziemlich grossen spindeligen Endothelzellen ausgekleidet sind, deren mittelgrosse Kerne mässig starke Tinction zeigen. An manchen dieser Lymphgefässe (Tafel VIII, Fig. 7) sehen wir, dass an der einen Seite, während die undere ganz normale Verhältnisse darbietet, die Zellen, die noch in einfacher Schicht liegen, bedeutend dickerund damit oval geworden sind, etwa fünfmal so breit als die normalen Endothelien der anderen Seite. Zugleich haben sich sowohl das Protoplasma als besonders die Kerne bedeutend stärker gefärbt. Weiter finden sich lange Lymphspalten, die wieder an der einen Seite vollkommen normales Endothel aufweisen, während sich hingegen an der anderen Wand an Stelle des normalen Belages eine zweifache Lage von Zellen vorfindet, die wesentlich nur durch ihre geringere Dimension sich von den oben erwähnten unterscheiden. Auch dreireihige Tumorzellenauskleidung findet sich. An anderen Stellen sehen wir 2-3und mehrschichtige, solide, von keinerlei normalen Elementen umkleidete, an beiden Enden zugespitzte, längliche Herde im Bindegewebe liegen. Hie und da ist auch eine perlschnurartige einreihige Tumorzellenkette zu sehen, die meist aus kleineren Zellen besteht, wodurch es wahrscheinlich wird, dass wir es mit Tangentialschnitten zu thun haben.

Die kleinen Cysten zeigen folgendes Bild. Bei schwaçer Vergrösserung (Tafel VIII, Fig. 8) sieht man, wie die Cystenwand ausgekleidet ist von einer Zellschicht, die von einschichtigen Stellen zu solchen mit starker Vielschichtung ansteigt, Die starke Vergrösserung. (Tafel VIII, Fig. 8a) zeigt, wie an einer Stelle dem Stroma ein einschichtiger Belag von spindeligen schmalen Zellen aufsitzt. Diese Zellen sind genau so beschaffen, wie die normalen Endothelien der Lymphgefässe und wohl auch mit diesen vollkommen gleichwerthig. Man sieht nun deutlich, wie an einer anderen Stelle die oben genannten Zellen zu grösseren, meh̀ kubischen Zellen von stärkerer Tinction, besonders der mittelgrossen runden Kerne werden, die ebenfalls dem Stroma direct aufsitzen. Diese letzteren fübren zu zweireihigem Belage der Cystenwand über, welche dann continuirlich in den vielschichtigen Tumorzellbelag übergeht.

Der Inhalt der kleinen Cysten besteht aus einer geronnenen, ganz homogenen, vor der Härtung serösen Masse mit einigen Leukocyten. Der Belag nach der grossen Cyste zu ist vielschichtig, mit unscharfer, offenbar öfters nekrotischer Oberfläche.

C. In der linhen Tube fällt das starke Oedem der Schleimbant auf, hie und da kleinzellige Infiltration derselben, der Musculatur and des Ligamentbindegewebes. Von Geschwulstbildung ist nichts zu seben.

D. Ein Schnitt durch den mittleren Abschnitt der hier etwas verdickten rechten Tube zeigt (Tafel VIII, Fig. 9), dass hier im Gegensatz zu links Geschwulstknötchen vorhanden sind. Zwei Knötchen liegen frei in cinem mit deutlichem Endothel ausgekleideten, blutleeren Ge- 
fässe, einem offenbar stark ektatischen Lymphraum. Ein drittes grösseres, kreisrundes Tumorknötchen findet sich in einer stärker aufgetriebenen Schleimhautfalte, die normale Epithelbederkung zeigt. Während die beiden ersterwähniten Geschwulstherdehen isolirt obne Zusammenhang mit dem Endothel in dem Hohlraum liegen, zeigt das letztere innigen Zusammenhang mit der Tubenschleimhaut, nur an einer kurzen Strecke wird seine Grenze gegen die Tumorschleimhaut gebildet von einem gestreckt halbmondförmigen Spaltraume (Tafel VIII, Fig. 10). Dieser Spaltraum ist nach der intacten Tubenschleimhaut zu von deutlichen; normalen, spindeligen Endothelien begrenzt. Auf der Tumorseite entsprechen diesen Zellen sehr grosse, gleichfalls spindelige, stark gefärbte Zellen, deren Leib theilweise gegen das Spaltraumlumen vorspringt. Da, wo der Spaltraum spitz ausgezogen endigt, gehen beide Schichten in eine über, die von einem zwischen beiden Zellarten die Mitte wählenden Zelltypus gebildet ist. An mehreren kurzen Strecken fehlen diese kalottenartig um das Tumorcentrum angeordneten endothelialen Elemente.

Alle Knötchen weisen die oben beschriebenen verschiedenen Arten von Tumorzellen, Riesenzellen, und Mitosen auf. Auffällig ist, dass sich auch um die beiden kleinen Geschwulstherdchen herum stellenweise grosse stark gefärbte spindelige Zellen finden, offenbar endotheliale Elemente. In der Umgebung der Tumorherde ist die Tubenschleimbant ödematös und zeigt Rundzelleninfiltration. Das Schleimhantepithel zeigt nirgends Zusammenhang mit dem Tumor.

E. An der hinteren Portiovaginalumsehlagsfalte geht das Tumorgewebe continuirlich in den später zu beschreibenden Tumor der Scheide über. Am Uebergang von Portio in den Cervicalcanal finden sich an beiden Lippen Erosionen mit starker rundzelliger Infiltration und starker hämorrhagischer Durchsetzung.

Im Gebiete des Cervicalcanales sind stark gewucherte Drüsen sichtbar und hie und da ein kleines Retentionscystchen; auch im Corpus, und hier sogar noch stärker, hat eine hochgradige Hypertrophie und Hyperplasie der Drüsen stattgefunden. Die Tumormassen sind grösstentheils alveolär angeordnet, in der hinteren Collumwand eng gedrängt, fast das ganze Gewebe einnehmend, nach oben zu mehr vereinzelt und mit grösserer Zerstreuung. Dort sind noch im oberen Drittel der hinteren Uteruswand hie und da kleine Tumoralveolen anzutreffen. Diese Alveolen sind zum Theil solide runde Zelicomplexe. Auch langgestreckte Zellzapfen finden sich, entsprechend dem derben Gewebe der Portio mit engen straffen Gewebsspalten, ziemlich häufig.

Hie und da sieht man kleine Lymphgefässe, die auf der einen Seite normales, auf der anderen Seite deutlich gewuchertes Endothel aufwiesen. Auch einreihige Tumorzellzapfen sind in Gewebsspalten ohne Endothelbekleidung vorhanden.

Die Tumorlumina sind theils Zerfallshöhlen, theils originäre Lymphräume und zeigen dieselben Arten der Abgrenzung gegen die Geschwulstzellen, die schon öfters beschrieben wurden.

Die stark differente Grösse der einzelnen Geschwulstherde ist auffallend. Die Stelle, wo die grössten Neoplasmaconglomerate zusammenliegen, findet sich am hintersten Theile der hinteren Wand der Cervix. Gegen den Cervicalcanal hin werden die Tumormassen spärlicher und zeigen weniger Nekrosen. An einer kleinen Stelle wird hier vom Tumor die Cervicalschleimhaut arrodirt und die Tumormassen grenzen frei an das Lumen des Cervicalcanales. Nur an dieser einen Stelle, wie an 
Serienschnitten nachgewiesen ist, grenzen Tumor- und Cervicalschleimhautzellen aneinander, wobei man aber nach Gestalt und Tinction beide Zellarten gut voneinander unterscheiden kann. Im übrigen ist die $\mathrm{Ab}$ grenzung gegen die erhaltene Scbleimhaut zu scharf; nirgends weist die Cervicalschleimhaut Tiefenwachsthum auf.

Die Beschaffenheit der Tumorzellen entspricht durchaus der schon ofter beschriebenen.

F. In der Vagina finden sich massenbaft Geschwulstherde. Die Tumorherde verhalten sich in Bezug auf Anordnung, Bau, Ahgrenzung gegen das Lumen wie oben beschrieben. Die Grenze gegen das sehr zellreiche und rundzellig infiltrirte Zwischengewebe ist nie scharf, sondern meist diffus. Die einzelnen Herdchen sind nicht von Endothel umgeben. Dagegen ist der Abschluss des Neoplasmas gegen das Lumen meist wieder von jenen vielerwäbnten spindeligen Zellen gebildet.

Das Scheidenepithel ist an manchen Stellen vom Tumor arrodirt in ganz ähnlicher Weise wie wir es an der Cervix gesehen haben, $d . h$. an den Stellen, wo der Tumor am weitesten nach dem Scheidenlumen za vorgedrungen ist, fehlt das Epithel, welches sich aber an der Grenze gegen die Geschwulstzellen genau so wie an intacten Stellen verhält, während die Tumorzellen sich in nichts von den schon oft beschriebenen unterscheiden. Das Scheidenepithel ist an manchen anderen Stellen bolzenförmig in gerader Richtung bis zu etwa dreifacher Dicke der normalen Scheidenschleimhaut in die Tiefe gewachsen, ohne sich jedoch in seinem Ansehen gegenüber den gesetzmässigen Epithelstellen verändert zu haben.

G. a) Ein Schnitt durch die entfernte rechte Drüse zeigt ein nicht sehr zellreiches, fettdurchsetztes, stark hämorrhagiscbes Bindegewebe. In ihm findet sich Lymphdrüsengewebe mit zahlreichen Geschwuistberden. Letztere sind meist solide, zum Theil sehr gut erhalten, zum Theil nekrotisch und zerklüftet, Die Abstände der einzelnen Zellen yon einander sind sehr verschieden, ebenso wie die Zellgrösse ziemlich wechselnd. Riesenzellen sind reichlich vorhanden.

In einer längs getroffenen Vene im paraglandulären Gewebe findet sich ein Conglomerat von nekrotischen Geschwulstzellen, auch Riesenzellen: dieser Tumorzellbalken ist von endothelialen Elementen umgeben und liegt frei im Lumen.

b) Die linke parametrane Drüse zeigt fast dieselben Verhältnisse. Hier erscheinen die Geschwulstzellen vielfach wie gemischt mit den adenoiden Elementen, so dass die Abgrenzung beider oft Schwierigkeiten bereitet.

H. Das bei der Fisteloperation (vgl. S. 191) zur Probe excidirte Stückchen, weist in einem stark hyalin und hydropisch degenerirten Grundgewebe stark zerkläftete unregelmässige Tumormassen auf. Auch einzelne zunde, kleine Geschwulstherde sind zu sehen, die sich gegen das Gewebe scharf abgrenzen, wobei jedoch weder normale Endothelien, noch endotheläbnliche Geschwulstzellen wahrgenommen werden. In den Gewebsspalten schieben sich zahlreiche ein- und zweireihige Tumorzellenstränge vor, deren Elemente genau so gestaltet siad, wie vorbeschrieben; ein etwaiger Belag aus gesonderten Endothelzellen ist auch hier niclut nachweisbar.

Fassen wir noch einmal alle Befunde in dem vorliegenden Falle zusammen, so ergiebt sich, dass wir es mit beiderseitigen. 
kugeligen, zum grössten Theil cystischen; zum kleinsten soliden Eierstockstumoren mit grosspapillären Excrescenzen und Scheidewänden im Innern zu thun haben. Im directen Zusammenhang mit dem rechten Ovarialtumor finden sich Tumormassen der hinteren Collum- und Scheidewand, Metastasen sehen wir in der rechten Tube und beiderseits in parametranen Drüsen.

Mikroskopisch finden wir den Tumor vorwiegend zusammengesetzt, einerlei an welchen Stellen die Untersuchung vorgenommen worden ist, aus alveolären und grosspapillären, theilweise soliden, teilweise mit einem Lumen versehenen Zellsträngen. An manchen Orten sieht man Spalten im Gewebe, deren eine Wand mit spindeligen endothelialen Elementen ansgekleidet ist, während die andere Wandseite eine Schicht von grossen ovalen Zellen aufweist. An auderen Stellen zeigt eine Wand wieder einschichtige Spindelzellen, während die andere Seite zwei oder mehr Lagen grösserer mehr ovaler Geschwulstzellen erkennen lässt. Auch perlschnurartige einzeilige Tumorzellenketten ohne Endothelumkleidung können wahrgenommen werden.

Die Wand der kleinen Cysten zeigt ron spindeligen einschichtigen endothelialen bis zu mehrschichtigen geschwulstmässigen Belägen alle Uebergänge. Das Cysten- und Alveolenlumen ist ausgefüllt mit einer homogenen Masse, die mit wenig Leukocyten, Detritus and Geschwulstzellen untermischt ist.

Infolge der im Vorigen festgesteliten Betheiligung der verschiedenen Genitalorgane an der Geschwulstbildung musste die kritische Beurtheilung zunächt den primären Erkrankungsherd festzustellen suchen. Die Annahme, dass der Uterus einen solchen beherberge, wurde schon bei makroskopischer Betrachtung des ungefärbten, noch mehr bei derjenigen des gefärbten Schnittbildes hinfällig. Schon die Ausdehnungsart der Geschwulst im Uterus, die so stattgefunden haben muss, dass sich die ersten Anfänge in der hinteren Wand am weitesten von der Schleimhaut des Cervixkanals entfernt bildeten, und von dort aus ein fächer- oder nierenförmiges Wachstkum nach der Schleimhaut zu stattfand, spricht gegen diese Annahme. Auch die Grössenverhältnisse der Ovárialtumoren gegenüber der Uterusgeschwulst erschweren eine derartige Auffassung. Eine primäre Uterusgeschwulst, ja noch mehr ein primäres Uteruscarcinon konnte also ausgeschlossen werdon; es ergab sich infolge dieser Ueberleguug, dass der primäre Sitz der Geschwulstbildung sich zweifellos im Eierstock befindet. 
Möglicherweise ist der rechte Eierstock, in dem die Tumormassen stärker gewuchert sind und in dessen Umgebung, besonders nach dem Uterus zu, dieselben sich weiter ausgebreitet haben, der Ausgangspunkt, wenn sich auch diese Frage nicht mit Sicherheit entscheiden lässt.

Es blieb nun noch die Frage nach der Geschwulstgattung zu beantworten.

In erster Linie musste an ein Kystadenoma papilliferum carcinomatodes gedacht werden; in der That schien anfangs das makroskopische und mikroskopische Bild diese Diagnose zu rechtfertigen, zumal bei der relativen Häufigkeit dieser Tumoren gegenüber der Seltenheit der Endotheliome. Ausserdem deshalb, weil viele Autoren überhaupt keine Endotheliome der Ovarien anerkennen. In der That liess zuerst das makroskopische und mikroskopische Bild diese Diagnose berechtigt erscheinen, denn wir haben es mit doppelseitigen cystischen Tumoren zu thun, mit zum Theil deutlich papillärer Structur, wobei mikroskopisch der alveoläre Habitus der Tumormassen und die atypische Zellproliferation auffällt.

Aber bald tauchten ernste Bedenken gegen diese Auffassung auf, und trotzdem die gegenwärtige Strömung in der Wissenschaft, unserer Ansicht nach in übertriebener Weise, vor derartiger Deutung warnt, stehen wir nicht an, in unserem Falle mit grösster Wahrscheinlichkeit ein Endotheliom anzunehmen. Es scheint uns an der Zeit, dass in dieser Beziehung die bekannte, der Hochflut stets folgende rückläufige Bewegung wieder einsetzt.

Die Diagnose eines Lymphangioendothelioms der Ovarien wurde eigentlich zum ersten Male mit Sicherheit 1879 von Marchand gestellt, nachdem schon vorher Leopold ein in diese Gruppe gehöriges "Lymphangioma cystomatosum" beschrieben hatte. Marchand erst wies sicher nach, dass es eine cystisch papilläre Geschwulst der Ovarien giebt, die von den Endothelien der Lymphgefässe abstammt, und dass man diese Art von Geschwülsten von den Sarkomen trennen müsse. Weitere Kenntnisse brachte dann über die endothelialen Geschwülste im Allgemeinen besonders Volkmann. L. Pick stellte 1894 drei Typen des Geschwulstwachsthums auf, die im Gegensatz zu Carcinom für Eierstocksendotheliome charakteristisch sein sollen. Es dreht sich bei Endotheliomen jedesmal um die Differentialdiagnose gegen Carcinom und als allein maassgebend werden meist nur die Anfangsstadien angenommen, daneben mehr oder weniger noch der Cysten- resp. 
Lichtungsinhalt, die Vielgestaltigkeit der Zellen, die Anwesenheit von Riesenzellen, die fliessenden Uebergänge in's Stroma. Auf die Möglichkeit, dass Lymphgefässe durch Erweiterung den Ursprung zu geschwulstmässiger Cystenbildung abgeben können, wies besonders Schottlaender hin. Die Endotheliome werden eben von den einen Autoren (Borst) als eine ziemlich gut charakterisirte Geschwulstart angesehen, während andere (R. Meyer) die Endotheliomdiagnose als so gut wie unmöglich hinstellen. Eine in jedem einzelnen Falle ganz sichere Unterscheidung von Carcinom und Endotheliom dürfte wohl auszuschliessen sein, doch kann wohl heute die Endotheliomdiagnose wenigstens mit grösster Wahrscheinlichkeit gestellt werden.

Die Frage nach der Stellung der mit den Endotheliomen eng verwandten Peritheliome, die besonders von v. Rosthorn, Amann, Rich. Krukenberg, Pollak behandelt wurde, ist noch nicht spruchreif, da der Begriff "Perithel" noch keineswegs consolidirt ist und darunter sehr versehiedene Elemente verstanden werden.

Gegen Carcinom spricht schon der ganze Bau unseres Tumors, der ja hier und da Andeutungen von mehr breit papillärem Warhsthum zeigt, ohne jedoch jene für das Adenocarcinom so charakteristische kleinpapilläre Structur mit durch das Zusammenwachsen der Papillenspitzen entstandenen Vacuolen darzubieten. In echten Carinomen sind die Papillen zierlicher, die Alveolen meist kleiner, die Grenze des Stromas gegen den Tumor immer stark ausgesprochen; das Zwischengewebe tritt mehr zurück. Ferner passen nicht zum Bilde des Carcinoms das vollkommene Fehlen eigentlich drüsenartiger Gebilde, der Reichthum an Riesenzellen, die Vielgestaltigkeit der Tumorzellen überhaupt, endlich der lymphatische Inhalt besonders der kleinen Cysten.

Für die endotheliale Herkunft unserer Geschwulst spricht zuerst die allgemeine formale Beschaffenheit derselben, indem von den drei von Pick aufgestellten Endotheliomtypen: 1. einreihige, rosenkranzartige Zellketten, 2. adenom- oder carcinomartige, lumenhaltige Schläuche oder solide Stränge; 3. diffuse sarkomartige Zellanhäufungen (die wohl sicher den Billroth'schen Alveolärsarkomen nahekommen), sich meistens die zweite Art vertreten findet. Es treten uns nämlich massenhaft Quer- und Längsschnitte von mehr oder weniger soliden, häufig schlauchförmigen Gebilden entgegen, die aus Tumorzellen zusammengesetzt sind. Doch auch der erste Typus kann öfters beobachtet werden, indem in Gewebslücken ein- 
reihige Zellketten gefunden werden, die von keinerlei endothelialen Elementen bekleidet sind.

Das Verhältniss des Neoplasmas zu den Lymphgefässen bietet einen weiteren Anhaltspunkt für die Endotheliomdiagnose. Die Erage über das Vorkommen der Endothelien (His. 1865) an Saftspalten und von Lymphgefässen im Ovarium ist bei der Betrachtung der Endotheliome (Golgi 1869) des Ovariums vielfach erörtert worden. Von His ist sicher nachgewiesen, dass das Letztere reichlich von Lymphgefässen und Saftspalten durehzogen ist; in Bezug auf die Auskleidung der Saftspalten gehen die Meinungen sehr auseinander. Borrmann nimmt eine sichere endotheliale Auskleidung auch der Saftspalten an, während Polano denselben kein Endothel einräumt. Jedenfalls kommen beide llodi vor. Im Ovarium sollen nach Injectionsversuchen keine Communicationen zwischen Lymphgefässen und Blutgefässen bestehen, ein Umstand, der die Deutung der Befunde Kolaczek's, der endotheliale Tumoren in Lymphräumen, Saftcanälchen und Blutgefässen gleichzeitig gesehen haben will, sehr erschweren würde. Auch eigentliche Perithelien lässt Polano nicht für dieses Organ gelten. Sie sind auch bis jetzt mit Sicherheit nur im Hoden, der Steiss-, Carotis-, Zirbel-, Brustdrüse und den Speicheldrüsen, sowie der Nebenniere nachgewiesen. Perivasculäre Lymphräume, deren Auskleidung von einigen Autoren (v. Rosthorn, Pick), ja auch als perithelial, ja von Borrmann als periendothelial bezeichnet wird, sind von Pollak im Ovarium sicher festgestellt. Aus dem Gesagten geht nur soviel hervor, dass es im Ovarium mit Endothel ausgekleidete Räume vermuthlich versehiedener Bedeutung reichlich giebt, die zum Ausgangspunkte einander sehr ähnlicher Tumoren werden können, wie ja auch die Pathologen Endo- und Perithelien als ziemlich gleichwerthig auffassen, worauf besonders Volkmann hinwies, während histoembryologisch das Endothel als dem Epithel näher gestellt betrachtet wird. Wie das ganze Bindegewebe in Tumoren neugebildetes Tumorstroma darstellt (Ribbert), so sind auch darin die Lymphgefässe nengebildet, was für die Eierstocksgeschwülste besonders Polano betont.

Wenn wir nun auf den Zusammenhang unseres Tumors mit. Lymphgefässen näher eingehen wollen, so muss vorausgeschickt werden, dass manche Forscher die Diagnose Endotheliom gerade im Ovarium als fast unmöglich hinstellen. So möchte Ribbert. Endotheliome nur dann sicher annehmen, wenn der fragliche Tumor 
in einem Organe gewachsen ist, wo normaler Weise Epithel nicht vorkommt, und auch keine Epithelverlagerungen bekannt oder wenigstens nach Lage der Verhältnisse ausgeschlossen sind. Auch R. Neyer macht auf die Forderung des fehlenden Zusammenhangs mit epithelialen Gebilden aufmerksam; ferner sollen die Tumorzellen in Reihen liegen mit Uebergang in diffuse und alveoläre Formen. Uebergangsbilder zwischen Geschwulstzellen und dicht benachbarten Endothelien sollen auffindbar sein, welch' letztere Forderung als berechtigt erscheinen muss.

Betrachten wir von diesen Gesichtspunkten aus unseren Tumor, so ist speciell im Ovarium eigentlich nie eine Versprengung epithelialer Elemente-ausgeschlossen, was aber viele Forscher (Flaischlen, Pomorski, Schottlaender) mit Recht nicht hinderte, sogar bei einem Dermoid eine endotheliale Neubildung anzunehmen. Ja Pfannenstiel hat sichere Combinationen von endo- und epithelialen Neubildungen gesehen. Bei der vorliegenden Geschwulst spricht nichts für die Entstehung aus epithelialen Elementen, vielmehr hat man wohl mit grosser Sicherheit den Ursprung des Tumors im Endothel der Lymphspalten und Lymphgefässe $\mathrm{zu}$ suchen.

Die Bilder, die zur Annahme dieses Ursprunges hauptsächlich Veranlassung gegeben, sind die besonders im Schnitt (B. 2) deutlich zu sehenden Stellen, wo an einem quer oder längs getroffenen Lymphgefässchen auf der einen Seite die Endothelien normal sind, d. h. spindelige, über die Fläche gekrümmte Zellen, mit länglichen stark tingirten Kernen darstellen, während auf der anderen Seite sich ein gewuchertes Endothel vorfindet, dargestellt von meist kubischen oder runden Zellen mit grossen mittelstark gefärbten Kernen ohne ganz deutliche Zellgrenzen. Hier ist kein normales Endothel zu finden. Die Bilder, bei denen sich (vgl. Fig. 7) auf der einen Seite normale Endothelien, auf der anderen grosse, weniger spindelige, aber immerhin endotheliale Zellen mit grossen stark tingirten Kernen finden, sind sicher als beginnende Tumorstadien, vielleicht als sich zur Theilung anschickende Zellen aufzufassen. Einfach reactive oder entzündliche Wucherung ist wohl nicht anzunehmen. Auch die ganz continuirlichen Uebergänge von den die kleinen Cysten auskleidenden spindeligen Elementen bis zu richtigen Tumormassen durch Stadien einschichtiger kubischer, zwei- und mehrschichtiger Beläge hindurch, sprechen für denselben Ursprung. Auf das vollkommene Fehlen einer endothelialen Aus- 
kleidung an den neoplastisch gewucherten Stellen als Kriterium des Endothelioms und differential-diagnostisch wichtig gegenüber Carcinom haben besonders Amann, Volkmann, Pick, Rich. Krukenberg hingewiesen. Glockner betont allerdings, dass ein Fehlen des Endothels ebensogut bei Carcinom beobachtet wird und auch Polano spricht von einer "Substitution des Endothels mit Carcinomgewebe", während Heinricius die Theorie aufstellt, dass ein Lymphangioendotheliom in den Lymphspalten beginnen könne, und dann ganz in der Art des Carcinoms in die Lymphgefässe hineinwuchere, welch letztere dann ihr Endothel behalten sollen.

Von manchen Seiten (Borst) ist auf die Schwierigkeit der Unterscheidung zwischen einfach entzündlichen gewucherten Endothelien, wie sie sich besonders beim Eintritt von Carcinomen in die Lymphräume und beim Fortschreiten in denselben finden, und solchen aufmerksam gemacht worden, die wirklich neoplastischen Wucherungen entsprechen. Auch Schottlaender spricht von einer einfachen Wucherung der Endothelien, im Gegensatz zu geschwulstmässiger Wucherung der Elemente, wobei zu beobachten ist, dass zwischen beiden Formen labile Uebergänge vorkommen, weshalb die Unterscheidung beider schwer, manchmal unmöglich ist. Aehnliche, wenn auch nicht dieselben Verhältnisse, nämlich einfache, d. h. nicht mehrschichtige und proliferirende Endothelwucherung beschreibt Kermauner bei Mittheilung eines Falles von Lymphangiom der Tube, wobei sich trotz im Wesentlichen einfacher Endothelwucherung doch anatomisch die kleine Geschwulst als entschieden maligne sich ausbreitend herausstellte. In unserem Falle gleichen die Zellen der oben beschriebenen Stellen so vollkommen denen in ausgesprochenen Tumormassen, dass von nur entzündlichen Processen keine Rede sein kann. Auch haben entzündlich gewucherte Endothelien nie die Gestalt jener grossen ovalen Zellen, die wir als das erste Stadium der Tumorentwickelung auffassten, sondern sie glichen mehr normalen Endothelien. Auch ist ihr Wachsthum regelmässiger und flächenhafter. Als differentialdiagnostisch wichtig gegen Carcinom stellt Borst noch das Fehlen einer Membrana propria an den schlauchartigen Alveolen hin, indessen ist auch bei Adenocareinomen die Membrana propria oft schwer und fast nur bei Anfangsstadien, meist aber gar nicht nachzuweisen, so dass uns dieses Kriterium nicht verwerthbar erscheint.

Um weiter die endotheliale Natur der Tumorzellen festzustellen, 
müssen wir uns mit der Anordnung der Tumorzellen und mit den verschiedenen Arten derselben, so wie sie uns besonders in den fortgeschrittenen Stadien der Geschwulst entgegentreten, sowie mit ihrer Abgrenzung gegen die später noch zu erörternden Iumina und gegen das Stroma beschäftigen.

Die endothelialen Geschwulstzellen sollen nach v. Hansemann während der Mitose von den carcinomatösen dadurch unterschieden werden können, dass die Endothelien besonders lange und schmale Chromosome, keinen Theilungsraum, endlich flache Spindeln mit sehr kleinen Chromosomen besitzen sollen. Uns ist bei Vergleichungen diese Unterscheidung nirgends geglückt. In den meisten Beschreibungen wird auf die epithelähnliche Beschaffenheit der Neoplasmazellen aufmerksam gemacht und besonders Borst weist auf die Metamorphose flacher endothelialer Elemente in kubische und eylindrische Zellen hin. Die Zellen in unserem Tumor sind meistens kubisch, polygonal. Ihre Wucherung geschieht durch einfache Mitose. Die Zellenabgrenzung verhält sich sehr verschieden; theilweise sind überhaupt keine Zellgrenzen zu unterscheiden, was von Schürmann, Rosinski, v. Velits ebenfalls angegeben wird. In neuerer Zeit hat man sehr häufig gerade endotheliale Syncytien gefunden (Schottländer, Schürmann) und Volkmann lässt derartige Gebilde durch Kerntheilung ohne Protoplasmatheilung entstehen.

Schottländer hält mit Anderen diese syncytialen Elemente in gewissem Sinne für endotheliale Wucherungen für charakteristisch.

Herz und Pollak geben an, dass die Zellen besonders in der Stromanähe pallisadenartig angelegt seien, eine Anordnung, die wir auch oft deutlich wahrnehmen konnten, während Rosinski von einer curvenartigen Aufstellung spricht.

Von besonderen Zellarten sind zunächst die Riesenzellen (Fig. 5) zu erwähnen, die sich reichlich in den Ovarialtumoren und in erstaunlicher Masse in den Tubenmetastasen vorfinden und über deren Bedeutung in endothelialen Neoplasmen nichts bekannt ist. Borst nennt ausdrücklich den gewöhnlich endothelialen Ursprung der Riesenzellen. In Endotheliomen werden Riesenzellen reichlich erwähnt, so z. B. von $\Delta \mathrm{mann}$, der gelappte Kerne beschreibt, von Pomorski, Voigt, Apelt, Federlin. Besonders Glockner hat das Vorkommen und die Eigenart der Riesenzellen in endothelialen Geschwülsten kennen gelehrt. Er stellt die Riesenzellen vollkommen gleich mit den anderen Tumorzellen. In unserem Falle 
liegen die Kerne, ca. 5-15, meist dicht zusammen, gewöhnlioh in der Mitte der Zellen und sind wohl durch Amitose entstanden; wenigstens gelang es nie, Mitosen festzustellen, doch sind letztere natürlich nicht auszuschliessen. Vielmehr siebt man bei den Riesenzellenkernen, dass diese entweder gleich gross und wohl durch directe Segmentirung (Arnold) oder seltener bei ungleicher Grösse durch Fragmentirung (Arnold) entstanden sind. Ein Analogon zu unseren einkernigen Riesenzellen (Fig. 4) bietet in der Literatur nur der Bericht Federlin's. Sollten diese grossen Gebilde Zellen darstellen, die vielleicht durch Fragmentirung oder Segmentirung ihrer Kerne demnächst zu mehrkernigen Riesenzellen werden? Dafür spräche auch der Umstand, dass man öfters eine Zelle mit zwei solch grossen Kernen findet.

Ferner sehen wir eigenthümliche, siegelringartige Zellen, bei denen durch hydropische Quellung des Protoplasmas der Kern an die Wand gedrängt und sichelförmig wird. Solche Zellen werden von Pomorski in seinen "sichelförmigen Zellen" und von Rosinski beschrieben. Diese Zellen sind jedenfalls genau analog den Siegelringzellen in Fr. Krukenberg's "Fibrosarcoma mucocellulare carcinomatodes", wo sie sich im Stroma und in Lymphräumen befinden und von manchen Autoren von den Endothelien abgeleitet werden.

Auch die hyalinen, schleimigen, amyloiden und hydropisehen Degenerationen (Borst) sowohl des Stromas als auch der Geschwulst sollen in gewissem Maasse für Endotheliome charakteristisch sein, wie dies ja auch von Pick, Volkmann, V. Rosthorn, Burckhardt erwäbnt wird. Hydropische und hyaline Degenerationen finden sich auch in unserem Tumor am Stroma und Neoplasma reichlich vor. Jedenfalls sind auch die in unserer Geschwulst zu beobachtenden, sich sehr stark mit Eosin färbenden Zellen mit kleinen Kernen weiter nichts als Zellen, die sich in beginnender hyaliner Degeneration befinden; auf dieses Verhalten in der Färbung machen Volkmann und Ernst aufmerksam. Hydropische Quellung findet sich reichlich an vielen Stellen des Stromas, in vielen der gewöhnlichen Tumorzellen und besonders bei den oben erwähnten Siegelringszellen.

Der Inhalt des Lumens ist in unserem Falle meistens aus Leukocyten, Detritus, einer offenbar geronnenen eiweissartigen homogenen Flüssigkeit und aus Tumorzellen zusammengesetzt, ganz so wie das Amann beschreibt. Auch der eigenthümliche Inhalt 
der kleinen Cysten, der offenbar aus geronnener lymphatischer Flüssigkeit besteht, spricht sehr für Lymphangioendotheliom. Wir baben es mit einer vollkommen homogenen Masse zu thun, die wie eine geronnene seröse Flüssigkeit aussieht und auch einige wenige Leukocyten enthält.

Die besonders von Leopold und Pomorski festgestellten "cellulären, zwiebelartigen Schichtungsbilder" als Lumeninhalt konnten wir nirgends nachweisen.

Während diese kleine Cystehen ziemlich sicher ektatische Lymphgefässe darstellen, lässt sich dies für die grosse Tumorcyste nur mit einiger Wahrscheinlichkeit annehmen; es sprechen in diesem Sinne die Befunde Schottlaender's, der plausibel gemacht hat, dass die meisten dermoidalen Cysten ursprünglich ektatische praeexistente Lymphräume darstellen, und darauf hinweist, dass dieser Modus der Cystenbildung überhaupt bedeutend häufiger vorzukommen scheint, als man vorher anzunehmen geneigt war. Wenm auch Borst cystische Lymphangioendotheliome als im allgemeinen seltene Tumoren betrachtet, und Volkmann betont, dass die Cysten nie von vornherein aus Lymph- oder Blutgefässen entstanden, sondern epithelialen Ursprungs sind, so sind doch schon eine ganze Reihe solcher Lymphcysten beschrieben worden. So vor allem von Marchand (1. Fall). Ferner halten Burekhard, Mirabeau, Sehürmann, V. Müller die bei ihren Fällen vorkommenden Cysten für Lymphcysten. R. Krukenberg endlich giebt an, dass Cysten sich häufig in Endotheliomen finden. Die Leistenvorsprünge an der Cystenwand sind wohl sicher die Reste zu Grunde gegangener Septen. Ausserdem sind als urspränglich vorhandene Cysten, deren Wand erst sekundär endotheliomatös entartete, beschrieben: Dermoide von Eckardt, Pomorki, Schottlaender, Schwertassek, Follikelcysten von Federlin, Cystadenome von Rosinski, Cystoma serosum simplex von Lange. Kworostansky endlich deutet einen Fall derart, dass neben dem Eondotheliome sarkomatöse und cylindromatöse Partien, Adenocarminomund Myomgewebe vorhanden sein sollen.

Papilläre Bildungen finden sich in unserem Tumor in dem Modus, den Marchand auffand, indem er angiebt, dass papilläre Formen der Lymphangioendotheliome nur da vorkommen, wo die endotheliale Wucherung grössere ektatische Lymphräume erfüllt. Borst beschreibt eystöse Bildungen mit papillärer Proliferation des Bindegewebes an den Wandungen der Cysten, die man sich offen- 
bar gerade so vorzustellen hat, wie die bei unserem Tumor beschriebenen papillären Partien.

$O b$ die in Fig. 6 dargestellten scharf durch endotheliale Zellen abgegrenzten runden Löcher praeexistente Lymphräume darstellen, lässt sich nicht sicher sagen, ist aber sehr wahrscheinlich, zumal auch bei Krukenberg-Tumoren, deren den Endotheliomen nahestehende Natur doch für einen Theil der Fälle angenommen wird, derartiges zu beobachten ist, wowei jedoch bemerkt werden muss, dass die Krukenberg-Tumoren keine einheitliche Geschwulstart verkörpern, sondern dass es darunter auch sichere Carcinome giebt. Sehr auffallend sind die Befunde an den Tubenmetastasen (Fig. 9 u. 10). bei denen sich am Rande von frei im Lymphraumlumen liegenden Herdchen sichere endotheliale Elemente finden. Diese sind nach unseren Anschauungen wohl so zu erklären, dass die Tumorzellen vielleicht infolge der Druckverhältnisse wieder zu ihrem endothelialen Charakter zurückgekehrt sind, wenn auch Endothelnenbildung nicht sicher auszuschliessen ist. Als direct versehleppte Endothelien sind diese Elemente wohl nicht aufzufassen.

An vielen Stellen ist der Tumor gegen das Lumen durch gradlinig nebeneinander liegende Zellen abgegrenzt, die sich durch nichts von den peripher gelegenen unterscheiden. Wir haben hier jedenfalls concentrisches Tumorwachsthum vor uns.

Da, wo wir Zerfallshöhlen vor uns haben, ist die Tumorlumengrenze naturgemäss ganz uncharakteristisch.

Meistens, nicht immer, liegt in der Umgebung des Lumens, sowohl an langen Spalträumen als auch an quergetroffenen Schläuchen zu sehen, eine Reihe von Zellen, die vielleicht etwas kleiner sind als die mehr excentrisch gelegenen Tumorzellen, deren Kerne länger oval, sehr stark gefärbt und mit ihrer längsten Achse circulär, resp. parallel zum Lumen geordnet sind. Für die starke Tinctionfähigkeit gilt wohl als ätiologisches Moment dasselbe, was Schottlaender für die in Sarkomen oft vorkommende Hyperchromatose annimmt, nämlich die besonders gute Ernährung, was gerade für die zunächst dem mit Lymphe angefüllten Lumen befindlichen Zellen besonders verständlich wäre. Die mehr platte Gestalt der dem Lumen anliegenden Zeilen liesse sich vielleicht auf die dem Drucke des Lymphstromas mehr ausgesetzte Lage der Zellen beziehen, wie ja überhaupt die Gestalt der Zellen fast nur von den äusseren Druckverhältnissen abhängig ist. Allerdings lässt sich nicht ausschliessen, dass wir in diesen Zellen noch 
am wenigsten veränderte Endothelien vor uns haben. Man müsste dann an diesen Stellen ein exentrisches Wachsthum des Tumors annehmen, womit sich sehr gut vereinigen liesse, dass die Geschwulst an anderen Stellen sich concentrisch ausgebreitet hat, zumal dieser doppelte Modus schon von Schottlaender und Schürmann beschrieben wurde. Das Endothel hat überhaupt eine sehr ausgesprochene Wucherungstendenz, wie schon aus Arnold's Versuchen hervorgeht, bei denen Fremdkörper von Endothel vollkommen umwachsen werden.

Ob die Richtung des Neoplasmas, nachdem das Endothel einmal den geschwulstbildenden Reiz empfangen hat, nach aussen oder innen stattfindet, hängt dann vielleicht wieder davon $a b$, $o b$ dor Lymphdruck den des Bindegewebes überwiegt oder umgekehrt. $\mathrm{Ja}$ es will scheinen, als ob die mehr erweiterten Lumina in Gegenden, wo das Bindegewebe zellärmer, vielleicht sogar hydropisch entartet ist, fast regelmässig von den oben beschriebenen Zellen eingefasst wären.

Das Stroma selbst weist viele hyaline und hydropische Degenerationen auf, ist von wechselndem Zellreichthume und eigentlich recht uncharakteristisch. Die Grenze zwischen Tumor und Stroma zeigt ein sehr wechselndes Verhalten. Oft findet man rom Tumor zum Stroma continuirliche Uebergänge, d. h. die Tumorzellen an der Peripherie sind nicht geradlinig gegen die Stromazellen abgeschlossen, sondern ihre Grenze stellt eine unscharfe Linie dar. Ferner sind die Tumorzellen in Bezug auf ihre mehr längliche Form und schwächere Tinction den Stromazellen noch ähnlicher als die mehr centralen Zellen. Auf diese Zustände machen besonders Leopold, Burckhardt, Fr. Krukenberg aufmerksam.

An einigen Stellen zeigt sich zwischen den Neoplasmazellen ein allerdings sehr spärliches Stromareticulum, welches von vielen (Borst) als charakteristisch für Tumoren der Bindegewebsreihe gehalten wird.

An anderen Stellen ist das Stroma rom Tumor scharf abgegrenzt, oft durch eine durch die Härtung bedingte Spalte. Andere Stellen erinnern an die Schilderungen Rosinski's, der die Alveolen nach aussen. gegen das Stroma von "normalem Endothel" umkleidet sein lässt, oder doch wenigstens theilweise, während an einzelnen Stellen diese Zellen vermisst werden. Ob diese längeren spindeligen Zellen mit stärker gekrümmten Kernen wirklich normale 
Endothelien darstellen, ist fraglich, ja im Hinblick auf die Thatsache, dass die peripheren Zellen, die am meisten dem Bindegewebsdrucke ausgesetzt sind, meist am plattesten sind, unwahrscheinlich.

Bei Betrachtung des Lumen- und Cysteninhaltes, des Fehlens von Blut in demselben, ferner des vollkommen unregelmässigen Verlaufes der Tumorzellenstränge, des Fehlens von Musculatur in der Tumorperipherie, der Intactheit der Blutgefässe - abgesehen von den hyalindegenerirten - muss wohl ein Lymphangioendotheliom als höchst wahrscheinlich angenommen werden mit Ausgang von den Lymphgefässen, wobei es sich nicht sicher unterscheiden lässt, ob dabei Saftspalten betheiligt sind oder nicht.

Ein Peritheliom liegt der ganzen Structur nach nicht vor, schon aus dem Grunde, weil nicht an allen Stellen intactes Endothel aufzufinden ist und weil an den Orten, wo wir als Lumenabgrenzung Endothel vor uns zu haben scheinen, sich nio eine irgendwie beschaffene Zwischenschicht $z$ wischen diesem und dem angenommenen peritheliomatösen Gewebe findet.

Als eigentliche Metastasen sind natürlich nur die Geschwulstherde in der rechten Tube und den beiden parametranen Drüsen aufzufassen. Bei dem grossen runden Herde in der rechten Tube allerdings ist eine selbstständige Entstehung - wie bei multicentrischem Tumorbeginne - wohl vorgetäuscht, da er mit fast seiner ganzen Circumferenz in der Tubenschleimhaut festsitzt; doch auch diese Vereinigung mit den Wänden eines sicher vorliegenden Lymphgefässes ist wohl secundärer Art; denn die rundo Gestalt, das calottenartige Umgebensein von endothelialen Elementen ist durch ein Entstehen dieses Herdes in der Tube selbst sehr schwer verständlich.

Die Tumorwucherungen im Uterus und in der Scheide bilden mit dem dicksten Theile des rechten Ovarialtumors dureh Verwachsungen ein Ganzes. Offenbar ist letzterer einfach in Scheide und Uterus eingewuchert. Auch ist der Geschwulsttypus in Uterus und Vagina derselbe wie im Ovarium. Uterusmetastasen sind nach Glockner selten, in der Scheide sind Metastasen noch seltener. Eine richtige Uterusmetastase bei Endotheliom beobachtete Federlin. Da keine echte Metastase vorliegt, so ist auch die Forderung, die Lange aufstellt, dass nämlich in Metastasen das Endothel erhalten sein soll, für die in Rede stehenden Geschwulstherde hinfällig. Dem raschen Wachsthum und der Ausbreitung im Uterus mag der reiche 
Gehalt an Saftcanälchen in letzterem Organe (Pick) zu Gute gekommen sein.

Wie meistens ist auch in unserem Falle der Stiel geschwulstfrei. Vergegenwärtigen wir uns den Weg, auf welchem es zur Metastasirung in den Tuben gekommen ist, so kommen wir als am wahrscheinlichsten auf die retrograde Verschleppung durch die Lymphbahn durch Umkehr des Lymphstromes bei. Verschluss einiger Hauptbahnen, der durch die Tumorbildung herbeigeführt sein kann. Doch wäre es auch denkbar, dass die Metastasen in der T'ube vom Uterus arsgegangen wären, entsprechend dem Lymphstrome, welchen Weg man dann auch für die parametranen Drüsen annehmen könnte. Der von Gebhardt, Martin, Olshausen angegebene Modus, nach dem die Metastase den Weg des Eies nimmt, ist ausgesehlossen, da die Tubenschleimhaut selbst unversehrt ist. Bei Capillarembolie (Virchow) wäre es sonderbar, dass gerade nur die Tube befallen sein sollte, ganz abgesehen davon, dass dieser Modus der Metastasirung für verhältnissmässig doch grössere Gebilde, wie Tumorzellen, noch gar nieht sicher erwiesen ist. Die Annahme einer "metabolischen Umwandlung" präexistenter endothelialer Elemente kommt jedenfalls auch nicht in Frage.

Bei den kleinen Tubenmetastasen ist ringsum die Endothelauskleidung der Lymphspalte gut erkennbar, was auch für eine Metastasirung auf dem Lymphwege spricht. Derselbe Modus der Verbreitung hat bei den Drüsenmetastasen stattgefunden. Wie gewöhnlich ist in den Drüsen das Bild ein vollkommen uncharakteristisches.

Interessant ist, dass sich bei unserem Tumor ein Befund zejgt, wie er auch von Lange beschrieben worden ist. Im Ligamentum latum findet sich, in der Nähe einer der oben genannten Lymphdrüsen, frei in einem Venenlumen ein Conglomerat ron zum Theil degenerirten Tumorzellen. In Ansehung dieses Befundes könnte man sich vorstellen, dass nach einem solchen Einbruche von Tumor in eine Vene auch eine Verschleppung auf der Blutbahn stattfindet, wofür sich aber in unserem Falle nirgends ein woitercr Anhaltspunkt findet.

Ueber die Aetiologie des Lymphangioendothelioms ist gar nichts bekannt.

Die klinische Betrachtung weist wenig dieser Tumorgattung Eigenthümliches auf, wenigstens ist es bis jetzt noch nicht gelungen ein für Endotheliome des Ovariums charakteristisches Krank- 
heitsbild aufzustellen. Ueber hereditäro Vorhältnisse ist auch in unserem Falle nichts bekannt. Gans übereinstimmend wird als bevorzugtes Alter die Zeit zwischen 40 und 50 Jahren, also die der cessatio mensium, angegeben, gerade wie es bei den Ovarialsarkomen der Fall ist. Nach Apelt fallen 20 pCt. aller Endotheliome in dies Alter. Ausserdem sind nach vielen Angaben Nullipare mehr von Endotheliomen befallen als Pluripare. Auch in unserem Falle haben wir es mit einer Nullipara zu thun. Meist ist der Beginn des Leidens mit Menstruationsanomalien verbunden, meist sind es Metrorrhagien oder, wie in unserem Falle, Menorrhagien, seltener plötzliches Ausbleiben der Menses.

Das Wachsthum unser Tumoren wird als sehr rasch geschildert. Die Geschwülste sollen schon innerhalb 6 Wochen kindskopfgross werden können. Damit geht Hand in Hand die enorme Malignität der Lymphangioendotheliome, die fast von allen Autoren anerkannt wird und die sich auch in unserem Falle durch das baldige Auftreten eines localen Recidives mit Ueberwuchern auf Blase und Mastdarm, sowic Fistelbildung gekennzeichnet hat. Auch das Verschwinden der Blasen- und Mastdarmfistel ist lediglich ein weiterer Beweis für die Wucherungsfähigkeit der Geschwulst. Die Schmerzen und Beschwerden weisen gar nichts Eigenthümliches auf und sind wohl meistens auf den zunehmenden Druck im Unterleib und Adhäsionen, auf Druckwirkung gegen Blase und Mastdarm zu beziehen. In unserem Falle ist die kurze Dauer der stärkeren Beschwerden - 3 Wochen - auffallend, während die Tumoren schon die angegebene Grösse erreicht hatten. Wieweit allerdings dabei die Indolenz der Patientin in Betracht kommt, lässt sich nicht sagen. Leichte Beschwerden, bestehend in stärkerer Periodenblutung, hatte Patientin bereits ein Jahr vorher gehabt. Doppelseitiges Auftreten der Geschwülste scheint bei Lymphangioendotheliomen nicht das Gewöhnliche zu sein. Nach Apelt werden nur in 22 pCt. aller Fälle die Tumoren doppelseitig beobachtet.

Entsprechend der Bösartigkeit dieser Erkrankung kann die Therapie nur in der Radicaloperation bestehen.

Um nochmals kurz zusammenzufassen, gründet sich unsere mit grosser Wahrscheinlichkeit zu stellende Diagnose "Lymphangioendothelioma ovarii" auf:

1. Die Bilder, bei denen Lymphräume an einem Theile der Wandung von normalem Endothel, an einem anderen von Tumorzellen ausgokleidet sind. 
2. Die gut zu beobachtenden Uebergänge der spindeligen Auskleidung der kleinen Cysten in Tumormassen.

3. Den Cysten- und Tumorlumeninhalt.

4. Die Vielgestaltigkeit der Zellen, besonders den Gehalt an Riesenzellen und syncytialen Elementen.

5. Die nicht deutlich papilläre Structur der Tumormassen, sowie das vollkommene Fehlen von eigentlich drüsigen Elementen.

6. Die oft fliessenden Uebergänge zwischen Stroma- und Tamorzellen.

\section{Li t e rat ur.}

1. Amann, Ueber Ovarialsarkome. Dieses Archiv. Bd. 46.

2. Derselbe, Mikroskopisch-gynäkologische Diagnostik. Wiesbaden 1897.

3. Apelt, Ueber die Endotheliome des Ovariums. Beitr. z. Geb. u. Gyn. Bd. V. 1901.

4. Borst, Die Lehre von den Geschwülsten. Wiesbaden 1902.

5. Borrmann, Zum Wachsthum und zur Nomenklatur der Blutgefässgeschwälste. Virchow's Archiv. Bd. 157. 1899.

6. Burckhardt, Cystische Ovarialtumoren endothelialer Natur. Zeitschr. f. Geb. u. Gyn. Bd, 40.

7. Eckardt, Ueber endotheliale Eierstockstumoren. Zeitschr. f. Geb. u. Gyn. Bd, 16.

8. Federlin, Ein Fall von Endothelioma ovarii mit Metastasen in Lymphdrüsen und Uteras. Hegar's Beitr. Bd. 8.

9. Flaischlen, Zur malignen Degeneration von Ovarialkystomen. Zeitschr. f. Geb. u. Gyn. Bd. 7.

10. Fränkel, Endothelioma ovarii. Monatsschr. f. Geb. u. Gyn. Bd.7.

11. Gebhard, Pathulogische Anatomie der weiblichen Sexualorgane. Leipzig 1899.

12. Glockner, Ueber das Vorkommen von ein- und mehrkernigen Riesenzellen und Riesenzellen mit Riesenkernen in endothelialen GeschwüIsten. Beitr. v. Ziegler. Bd. 26. 1899.

13. Gräfe, Zwei Fälle von Endothelioma bezw. Perithelioma ovarii. Ein Fall von Endotheliom der Port, vag. Dieses Archiv. 1904. Bd. 72.

14. Guffroy, Endothelioma ovarii. Inaug.-Diss. Freiburg, Amsterdam 1897.

15. Heinricius, Ein Fall von Endothelioma lymphaticum ovarii. Dieses Archiv. Bd. 73. 1904.

16. Herz, Zur: Kenntniss des Endothelioma ovarii. Monatsschr. f. Geb. u. Gyn. Bd. 9. 1899.

17. Jung, Gabrie], Zur Diagnose und Histogenese des Ovarialcarcinoms. Beitr. z. Geburtsh. u. Gyn. 12. Bd. 3. H.

18. Kaufmann, Lehrbuch der speciellen Pathologie. Berlin 1907. 
19. Kr etschmar, Endothelioma ovarii cysticum. Verhandl. d. 9. Gyn.-Congr. 1901.

20. Krakenberg, Fr., Ueber das Fibrosarcoma ovarii mucocellulare carcinomatodes. Dieses Archiv. Bd. 50.

21. Krukenberg, Richard, Beitrag zur Kenntniss des Perithelioma ovarii. Zeitschr. f. Geb. u. Gyn. Bd. 41.

22. Lange, Ein Fall von Endothelioma ovarii. Centralbl. f. Gyn. 1903.

23. Leopold, Die soliden Eierstocksgeschwülste. Dieses Aroh. Bd.6.

24. Marchand, Beitrag zur Kenntniss der Ovarientumoren. Habil.-Schr. Halle 1879.

25. Meyer, R., Das Endotheliom des Uterus. Veit's Handb. d. Gyn. Wiesbaden 1908.

26. Mirabeau, Perithelioma ovarii oystioum. Monatsschr. f. Geb. u. Gyn. Bd. 10.

27. Müller, V, Ueber Carcinome und Endotheliome des Eierstocks. Dieses Archiv. Bd. 42.

28. Pfannenstiel, Die Krankheiten des Eierstocks. Veit's Handb. d. Gyn. Wiesbaden 1898.

29. Pick, L., Von den Endothelien ausgehende Geschwülste des Eierstocks. Berl, klin. Wochenschr. 1894. No. 45 и. 46.

30. Derselbe, Zur Lehre vom Myoma sarcomatosum und über die sog. Endotheliome der Gebärmutter. Dieses Archiv. Bd. 49.

31. Polano, Ueber Pseudoendotheliome des Eierstocks. Zeitschr. f. Geb. u. Gyn. Bd. 51. 1904.

32. Pollak, Zur Kenntniss des Perithelioma ovarii. Monatsschr. f. Geb. u. Gyn. 1907.

33. Pomorski, Endothelioma ovarii. Zeitsehr. f. Geb. u. Gyn. Bd. 18.

34. Rosinski, Zar Lehre von den endothelialen Ovarialgeschwülsten. Zeitschrift f. Geb. u. Gyn. Bd. 35.

35. v. Rosthorn, Zur Kenntniss des Endothelioma ovarii. Dieses Archiv. Bd. 41.

36. Schottlaender, Zur Lehre von den Dermoideysten des Eierstockes. Dies. Arch. Bd. 78. 1906.

37. Schürmann, Ein Fall von Endothelioma ovarii lymphaticum cysticum. Zeitschr. f. Geb. u. Gyn. 1903. No. 50.

38. Schwertassek, Sarkomatöse Degeneration einer mit einem Teratom combinirten Ovarialcyste. Dieses Arch. No. 47.

39. v. Velits, Endothelioma cysticum myxomatodes ovarii. Zeitschr. f. Geb. u. Gyn. No. 18.

40. Voigt, Ein Fall von Kaiserschnitt nach Porro in der Schwangerschaft wegen malignen Ovarialtumors nebst Beitrag zur Pathologie des Corpus luteum. Dieses Arch. Yo. 49.

41. Derselbe, Zur Kenntniss des Endothelioma ovarii. Dieses Arch. No, 47.

42. Volkmann, Ueber endotheliale Geschwülste, zugleich ein Beitrag zu den Speicheldrüsen und Gaumentumoren. Deutsche Zeitschrift für Chirurgie. Bd. 41. 1895.

43. Ziegler, Lehrbuch der allgemeinen Pathologie und der pathologisehen Anatomie. Jena 1901. 
44. Kermauner, Lymphangiom der Tube. Dieses Archiv. Bd. 83 .

45. Kworostansky, Endotheliom des Orariums und der Tube. Dies. Arch. Bd. 85 .

\section{Erklärung der Abbildungen auf Tafel VII-VIII.}

Figur 1. Rechter Ovarialtumor, aufgeschnitten. Septen. In der Mitte Uebergang in die Nebencyste. Grosspapilläre Structur. Kleine Cystchen in der Wand.

Figur 2. Uterus-Sagittalschnitt. In der hinteren Collumwand, nierenförmig ausgebreiteter Tumor, der auf diesem Schnitte die Schleimhaut nicht berührt.

Figur 3. Längsschnitt aus einer mitteldicken Wandstelle der grossen Cyste des rechten Tumors. Nach unten z. Th, einschichtiger Tumorbelag der grossen Cyste. Kleine und grosse Alveolen, die Lumina letzterer z. Th. Zerfallshöhlen darstellend. Hier und da Tumorzellbrücken die Lumina überspannend.

Figur 4. Grosse (Riesen-) Zellen mit einem oder zwei grossen Kernen zwisehen gewöhnlichen Tamorzellen.

Figur 5. Riesenzellen mit vielen, theils gleich-, theils ungleich grossen Kernen.

Figur 6. Siegelringartige Zellen und Hohlräume, die von endothelialen Elementen umgeben sind.

Figur 7. Schnitt aus der Wand einer kleinen Cyste, die an dieser Stelle mit unveränderten Endothelelementen ausgekleidet ist. Im Stroma zunächst normale Lymphräume, ferner solche, die an der einen Wandseite normale Elemente und an der anderen grosse gequollene Zellen tragen, weiter Lymphgefässe, die neben normalen Wandstellen solche mit ein-, zwei- oder mehrschichtigem Tumorbelage ansgekleidet zeigen, endljch perlschnurartige Tumorzellketten.

Figur 8. Cystenauskleidung. Schwache Vergrösserung. Uebergang vom normalen Endothel in Tumorzellbelag.

Figur 8a. Dasselbe bei starker Vergrösserung.

Figur 9. Tubenquerschnitt. Zwei frei in ektatischen Lymphspalten liegende Tumorherdchen mit Riesenzellen. Eine Tubenfalte, sehr aufgetrieben, einen kreisrunden Tumorherd enthaltend, der nach unten zu durch eine sichelförmige Spaltbildung ron der Tubenschleimhant getrennt ist.

Figur 10. Letzteres Bild vergrössert; nach unten, nach der Tubenschleimhaut zu nurmales Endothel, nach oben zu grosse gequollene endotheliale Tumorzellen. 
\&
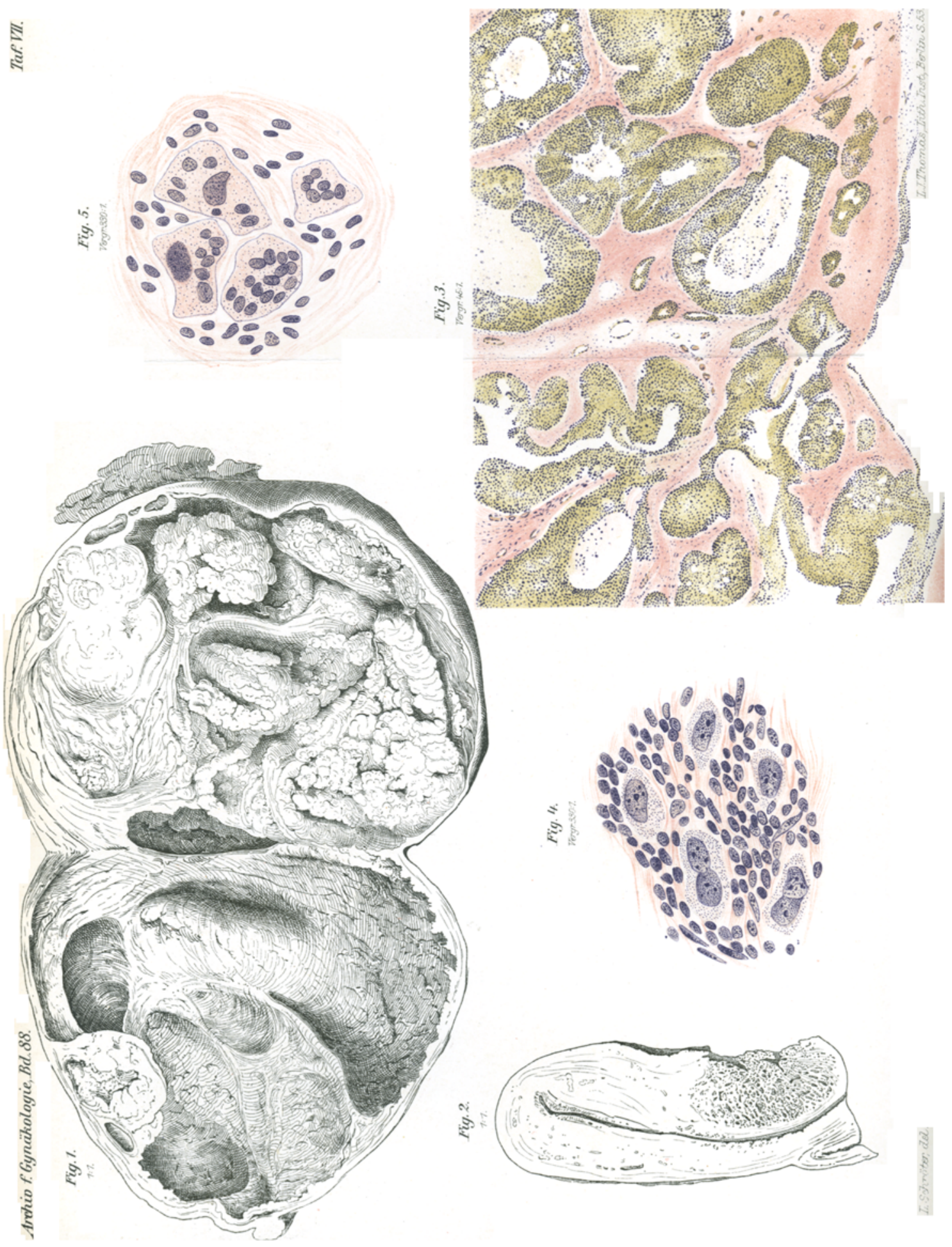

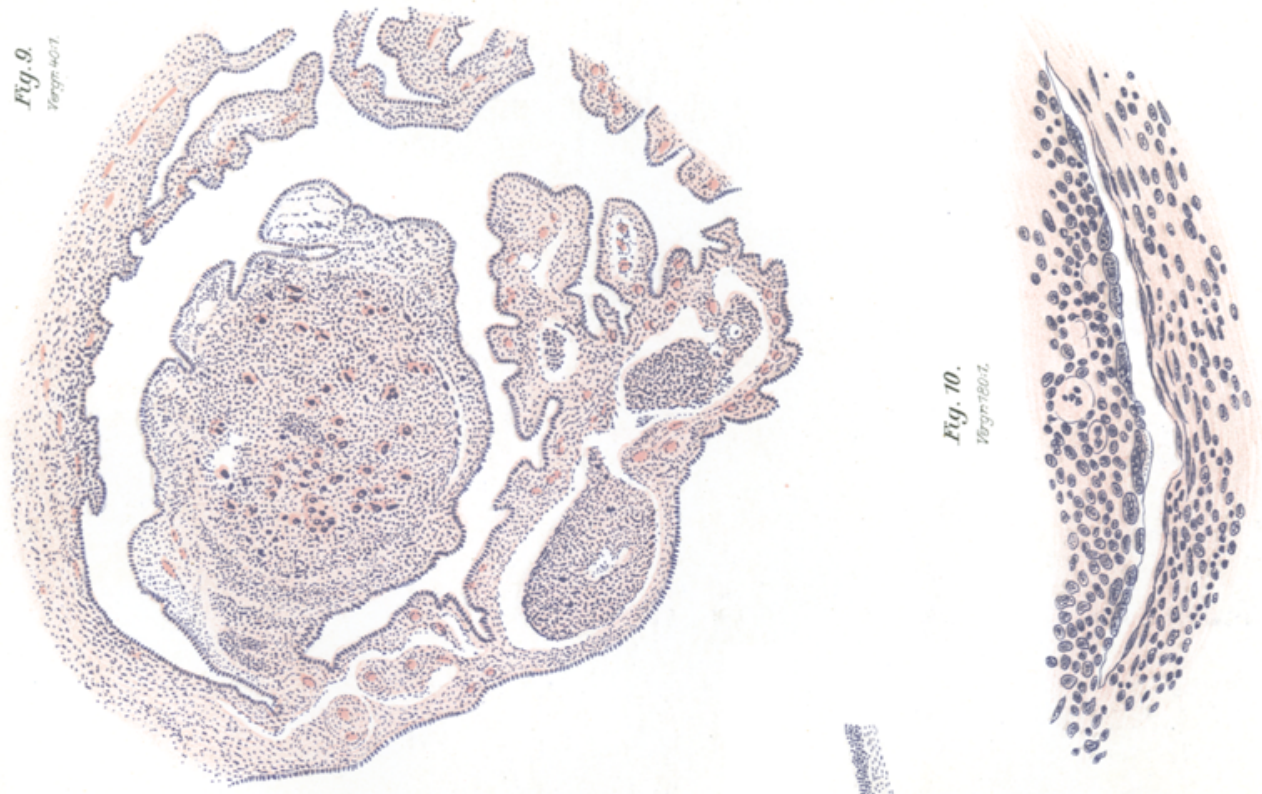

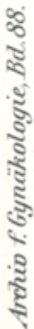

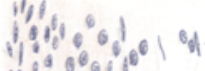

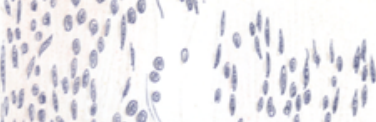

$$
\begin{aligned}
& \text { (11979, is }
\end{aligned}
$$

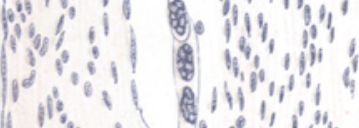

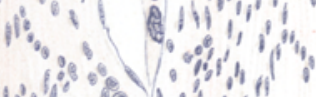
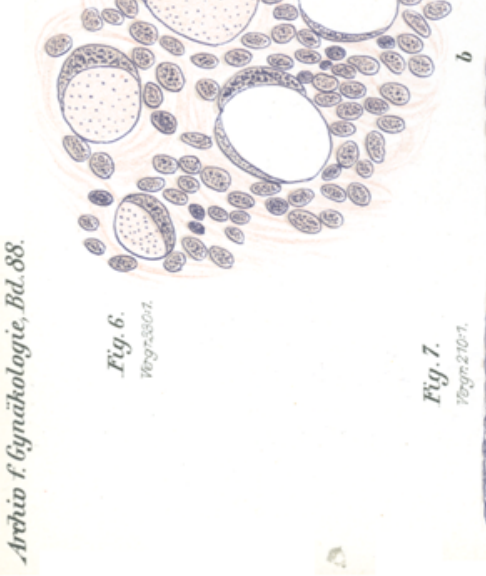

ฟ่ำ

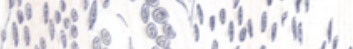

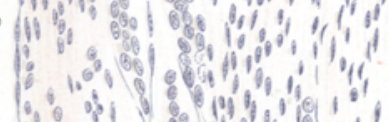

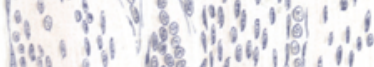

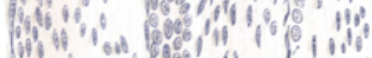
"

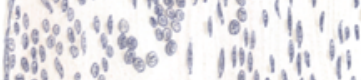

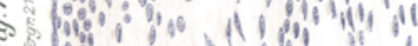

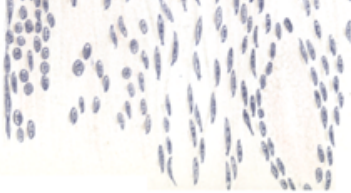
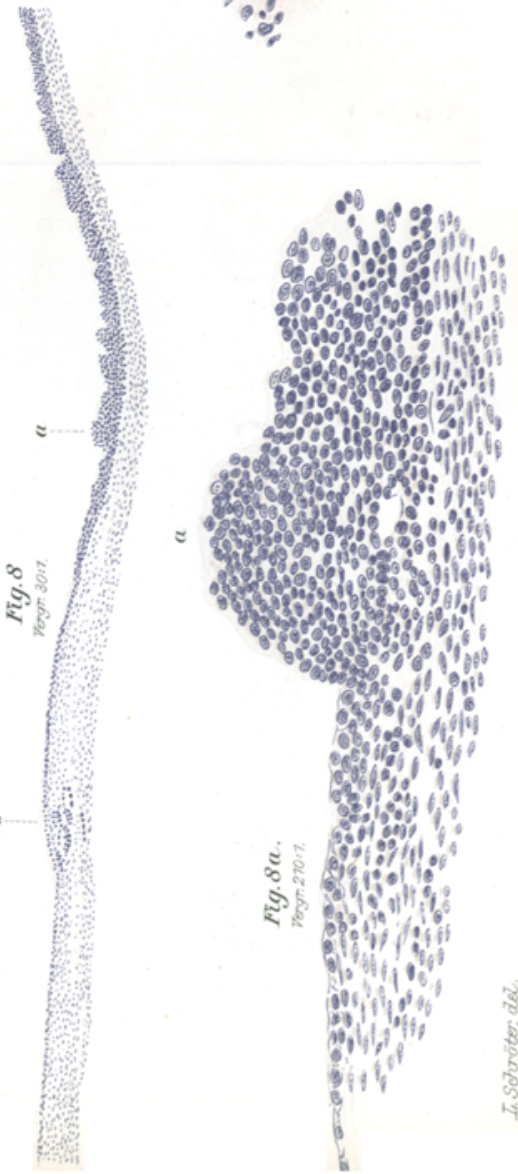